\title{
CONSIDERACIONES GENERALES SOBRE POBLACIÓN, DESARROLLO Y MEDIO AMBIENTE, EL CASO DE TIJUANA, BAJA CALIFORNIA
}

\author{
Por \\ Margarita Barajas Tinoco* \\ Elizabeth Méndez Mungaray**

\section{RESUMEN}

A partir de las relaciones que se pueden encontrar entre la sociedad y el medio ambiente natural, en este trabajo se destacan los elementos que dentro de la planeación general se orientan a la población y el desarrollo. El concepto de "calidad de vida", en sentido amplio, es tomado como indicador de desarrollo para problematizar contradicciones manifiestas entre un crecimiento urbano particular y su déficit de infraestructura social y problemas de medio ambiente. Como estudio de caso ilustrativo se toma a la ciudad de Tijuana, espacio territorial que enfrenta una problemática urbana, manifiesta entre otros escenarios, en su medio ambiente natural y social.

\begin{abstract}
From the point of the relations that can be found between society and the natural environment, this work emphasizes the elements that, in general planning are orientated to in a broad sence, population and development. The concept of "life quality" is taken as an indicator of growth to problematize manifest contradictions within a particular urban increase and its social infrastructure deficit and enviromental problems. The city of Tijuana is taken as an ilustrated case of study, territorial space that faces a urban problem, manifested, among other scenaries, in its social and natural environment.
\end{abstract}

\section{INTRODUCCIÓN}

El trabajo que se presenta consta de tres apartados, en el primero se maneja la relación sociedad-naturaleza y su objetivo es destacar las interacciones complejas que este binomio presenta en la actualidad; se tiende a destacar la relevancia de los procesos naturales en el desarrollo y progreso del hombre a través de la conciencia de su medio ambiente. En el mismo sentido, el subapartado sobre población y desarrollo marca de forma concreta el proceso de esta relación y su manejo desde el ámbito

* Investigadora del Instituto de Investigaciones Sociales de la UABC.

** Investigadora del Departamento de Estudios Urbanos y Medio Ambiente de El Colegio de la Frontera Norte. 
público. En el segundo apartado se describen de manera general algunas características relacionadas con el proceso de urbanización de la fronteranorte de México (FNM), a través de la documentación sobre su población y economía. Este apartado se toma como marco referencial en donde se ubica más detalladamente el caso de una ciudad fronteriza: Tijuana, Baja California.

El objetivo del tercer apartado es dar sentido y significado a los conceptos manejados en la primera parte del trabajo. Mediante la descripción del proceso de conformación y crecimiento de la ciudad de Tijuana. Con ello se destacan los principales problemas derivados de la relación ciudad-medio ambiente y se toca un grupo de elementos que en su conjunto tienen que ver con la calidad de vida de la población de esta ciudad fronteriza.

El objetivo global de este trabajo consiste en la elaboración de un marco de referencia cuyo ulterior desarrollo encauce un tipo de investigación socio-espacial, partiendo de la consideración de que un conglomerado social puede ser visualizado en términos de dos categorias: su ambiente biogeofísico y su ambiente social; ambientes que a su vez definen la calidad de vida de la sociedad.

\section{MARCO GENERAL: ARTICULACIÓN SOCIEDAD-NATURALEZA}

La concepción sociedad-naturaleza nos obliga a la búsqueda de una conceptualización que pretende ser globalizadora. Los procesos de desarrollo de una comunidad, ciudad, estado o país, no es posible concebirlos como independientes de su medio físico-biológico, así como tampoco dicho medio natural puede concebirse como espacio no explorado, sin destacar la función principal que proporciona al hombre a través de los recursos naturales y medio ambiente que genera.

El nexo que podemos encontrar entre sociedad y naturaleza se produce a través de dos tipos de factores: El conjunto de acciones humanas que inciden sobre el sistema ecológico natural (es decir, sobre el sistema estructural y funcional de la naturaleza), y el conjunto de efectos ecológicos generados en la naturaleza y que inciden sobre el sistema social (Gallopín, 1986:162). Los efectos ecológicos son las respuestas de los sistemas naturales a las acciones humanas y que finalmente van a conformar un tipo de medio ambiente. A su vez, en esta relación sociedad-naturaleza, el medio ambiente puede ser conceptualizado como "todo aquello que rodea al ser humano y que comprende a los elementos naturales, tanto físicos como biológicos, a los elementos artificiales (tecnoestructuras), a los elementos sociales y a las interrelaciones de todos entre si" (Sánchez, 1982).

Hasta aquí, tenemos que la relación sociedad-naturaleza es una relación directa que va en ambos sentidos y que, dependiendo cómo sea su contenido, 
conforma un determinado medio ambiente. Por tal razón, las características ambientales en general pueden diferir entre personas o grupos dentro de una misma sociedad, y también entre sociedades. Insistiendo sobre la concepción de medio ambiente y entendiéndola como el resultado de la interacción sociedad-naturaleza, es importante ampliar la explicación de su concepto. El ambiente de un conglomerado social puede ser visualizado en términos de dos grandes categorías: por un lado, el ambiente biogeofisico-químico y, por otro, el ambiente social. El primero incluye componentes como el aire, el agua, el clima, el campo o paisaje, las condiciones de higiene, las condiciones físicas de vida, plantas y animales, etcétera. En cambio, el ambiente social de las personas y grupos incluye factores tales como cl tipo y calidad de las relaciones interpersonales o intergrupales, el acceso al trabajo productivo, el acceso a la educación y a la cultura, las condicionantes externas de la participación y libertad de expresión, las influencias psicosociales, entre otras (Gallopín, 1986:153). Esta concepción de medio ambiente, o más bien de ambiente humano, considera relaciones entre los hombres, entre los hombres con las cosas (su medio físico circundante) y las relaciones de las cosas entre sí. El grado de complejidad que puede adquirir el manejo conceptual de estos diversos componentes obliga, y no está por demás decirlo, a una interdisciplinaridad de conocimientos sobre un mismo objeto de estudio.

Un concepto que es necesario definir para completar el esquema pertinente al complejo sociedad-naturaleza, es el de "calidad de vida". El ambiente humano supone de entrada ciertas condiciones ambientales que son inherentes a cualquier conglomerado social; estas condiciones, según se manifiesten y caractericen, se relacionan y explican (en la medida que definen) la calidad de vida. Dicho concepto es concebido como:

la resultante de la salud psicosomática de una persona (evaluada objetiva o intersubjetivamente) y del sentimiento (subjetivo) de satisfacción. La salud depende de los procesos internos de la persona y del grado de cobertura de los deseos y aspiraciones. Mientras que las necesidades humanas son generalmente concebidas como requerimientos genéricos de los seres humanos para mantenerse saludables, los deseos y aspiraciones son las formas concretas en que una persona busca cubrir sus necesidades percibidas, denotando los satisfactores específicos requeridos (Gallopín, 1986:158).

Esta conceptualización de calidad de vida nos da la pauta para considerar una serie bastante amplia de condiciones y situaciones que provocan un determinado nivel de calidad de vida adjudicable al conglomerado 
social, no obstante, para efectos del presente trabajo, el concepto al que se alude tiene que ver más con la calidad del espacio ambiental y construido, que con los indicadores de empleo, ingreso o escolaridad que más usualmente se toman en cuenta. Si bien, estos deben guardar algún grado de correspondencia con la ocupacion y calidad del espacio de los individuos, en este trabajo, planteado a nivel general, pasan a segundo término. En todo caso, serían fundamentales al momento de plantearse la medición de un índice al respecto de la calidad de vida en una o más zonas espaciales, metodológicamente delimitadas de la ciudad. ${ }^{1}$

Una vez que completamos el esquema básico de los elementos interactuantes entre sociedad y naturaleza podemos problematizar la relación de una forma bastante general. Por ejemplo, actualmente, en un mundo de más de 5 mil millones de habitantes, donde el desarrollo tecnológico aplicado a los procesos productivos de la vida moderna repercuten seriamente sobre el entorno natural, la relación entre sociedad-naturaleza se ha vuelto compleja y en muchos grados irracional. El proceso de influencia del hombre sobre su medio ha adquirido características que han roto con los sistemas naturales de procesos armónicos de la vida vegetal, animal, atmosférica, etcétera.

Lo que en un momento se entiende como medio ambiente, después se maneja como problemática ambiental, que no es más que el conjunto de resultados obtenidos del agravamiento de los problemas derivados de la forma irracional de uso de los recursos, además de los problemas derivados en tomo a la contaminación y sus secuelas en la salud del hombre. En sí,

la presión sobre el medio ambiente ha aumentado progresivamente y las formas de esta presión se han multiplicado, debido fundamentalmente al crecimiento de la población, a su desigual distribución y acceso a los recursos; a las características de los sistemas socioeconómicos de utilización del medio ambiente y a las características propias de la tecnología moderna (Ojeda y Sánchez, 1984:2).

Uno de los enunciados fácilmente cuestionado es que el deterioro del medio ambiente no es más que el costo del desarrollo y progreso; si bien el enunciado encierra una gran verdad, resulta parcial. En primer lugar, parece que da la pauta a un inmovilismo, a una resignación que acepta ya toda inercia ante la degradación del medio ambiente; por otra, el enunciado es parcial en la medida que deja de asumir que los efectos más graves sobre

1 Un trabajo de investigación así definido, cuyas variables son de naturaleza socioespacial, se piensa como continuación del aquí expuesto. 
el medio ambiente provienen también de los países y regiones más industrializados de la tierra, así como tampoco observa que los resultados y repercusiones del medio ambiente distorsionado inciden más sobre las regiones y grupos sociales menos desarrollados. Se supone, pues, que se tenderá y alcanzará el desarrollo y progreso, aun a costa de cierto deterioro ambiental. Hay que tener presente que el mismo deterioro ambiental llevado al extremo pone en serio peligro el cumplimiento de los objetivos del desarrollo. Es por ello también que la interacción sociedad- naturaleza, dada en condiciones irracionales, no implica mas que repercusiones directas e indirectas que pueden afectar el bienestar de la población. ${ }^{2}$

Tomando en cuenta que el deterioro del medio ambiente se debe en gran medida a la intervención del hombre sobre la transformación de la naturaleza y a los objetivos de desarrollo, se cree estar ante una disyuntiva que plantea que: o se alcanza el desarrollo o se conserva el medio ambiente. Esta disyuntiva es extrema y no tiene razón de ser, aceptarla de entrada significaría no reconocer todas las potencialidades de la naturaleza en nuestro mundo contemporáneo, sobre todo en países como el nuestro, donde se tiene la necesidad de aprovechar lo que el medio natural ofrece. ${ }^{3}$ En este sentido lo deseable es un desarrollo sustentable ${ }^{4}$ encaminado a lograr un crecimiento con equidad y protección del medio ambiente.

Para finalizar este apartado, cabe agregar que, bajo la aceptación de complejidad de la relación entre el binomio sociedad-naturaleza, es necesario

2 En relación a esta problemática se ha mencionado que esta modalidad o estilo prevaleciente de desarrollo ha estado vigente hasta la fecha, considerando a la naturaleza como si fuera inerte, desconociendo en consecuencia que la misma se trata de un complejo "sistema de sistemas en interaccion", extraordinariamente vivo y del cual el hombre es parte integrante, en tanto ser biológico. Los mecanismos propios que le han permitido a la naturaleza regenerarse, producir diferencias, absorber y reciclar elementos (desechos), están siendo sobrepasados por la presión ejercida sobre ella y de allí el deterioro ambiental mencionado (Ojeda y Sánchez, 1984:3).

Por ejemplo, planear la ciudad en concordancia con los procesos ecológicos naturales e incorporando la importancia de la vegetación en el paisaje urbano, proporcionaría al hombre un ahorro considerable de energía y por lo tanto de costos. Tal es el caso de un árbol que individualmente puede transpirar 100 galones de agua por día, lo cual equivale a $230 \mathrm{mil}$ kilocalorías de energía en evaporación. Esta energía se la ahorra la ciudad en el intento de controlar la temperatura de la paredes de edificios y pavimentos por otros medios, pues el equivalente mecánico para la evaporación de ese árbol (100 galones de agua es, en promedio, el uso de cinco aires acondicionados, que gastan 2,500 kilocalorías por hora y funcionan 19 horas al día) (Cuttler y Cutler, 1982).

4 Los objetivos tradicionales de desarrollo están orientados a satisfacer las necesidades básicas de la población y en aumentar la productividad de todos los recursos; un desarrollo sustentable, además de esto, tiene que conciliar "la existencia y preservación, para futuras generaciones, de condiciones ecológicas necesarias para mantenerla vida humana en un nivel específico de bienestar" (Lele M., 1991). 
adscribirse a metodologías y marcos teóricos que garanticen el análisis y estudio de un enfoque global, donde la realidad no sea fraccionada en una variedad de disciplinas y conceptos únicos. En realidad, la sociologia, demografía y la ecología son las áreas del conocimiento que están dando la pauta para la conformación e integración de un cuerpo sólido de categorías y conceptos para abordar el estudio sobre la sociedad y su medio ambiente. ${ }^{5}$

\section{Población y desarrollo}

Actualmente existe un reconocimiento en diferentes ámbitos sobre la relación entre la población y su grado de desarrollo alcanzado. Se ha aceptado que las políticas, planes y programas de planificación deben considerar a la población como la base de dónde partir y el objetivo para incidir en el logro de resultados que se consideran como idóneos. En la declaración de la ciudad de México sobre población y desarrollo (CONAPO, 1984a) se señala que se debe dar prioridad a los programas de acción que integren todos los factores básicos de población y desarrollo y que tengan plenamente en cuenta la necesidad del aprovechamiento racional de los recursos naturales y la protección del medio ambiente físico para evitar que siga deteriorándose.

En el mismo sentido, el Programa Nacional de Población (PNP) 19891994 (CONAPO, 1991a), establece que la población es simultáneamente el recurso básico para el desarrollo y su beneficiario; a partir de dicha concepción se deben de desprender todas las acciones contempladas en el PNP en coordinación con el resto de las autoridades del sector público involucradas. Hasta aquí, se puede inferir que la estrategia fundamental para encaminarse al desarrollo está puesta en la planeación en todos niveles, por ello es pertinente apuntar dos premisas que la planeación del desarrollo debe considerar en los estudios sobre población.

1. La población y sus características dependen del desarrollo económico y social, por lo tanto, no se puede analizar de maner aislada circunscrita exclusivamente a un centro de población para ser tomada como unidad cerrada, sino estudiada a la luz de las características económicas del centro de población respecto al desarrollo económico regional. Esto supone que la dinámica demográfica de un centro de población es imposible de entender si no se precisa y entiende su contexto.

5 Al respecto ver comentarios del seminario "Población y ambiente", organizado por la Sociedad Mexicana de Demografía (someDE, 1992) y de Fernando Tudela el artículo "Población y medio ambiente" (1991). 
2. Por otro lado está la referencia a la determinación de orden económico, toda vez que constituye el factor dominante en la vida social a la vez que condensa la dinámica política. Resulta necesario considerar la dinámica política como una heterogeneidad de sujetos políticos y en cuanto tales, con posibilidad para modificar las determinantes económicas. La planeación, en tanto que pretende transformar la realidad, se encuentra también dentro del contex to sociopolítico del cual la población forma parte activa. ${ }^{6} \mathrm{El}$ contenido de estas premisas nos deja ver que la relación del binomio que estamos manejando no se da de forma unilateral; no existe una relación única entre sujeto y objeto, todo lo contrario, la población como factor impulsador del desarrollo es a su vez afectado diversamente por éste, así vemos que él mismo, es decir, el nivel de desarrollo, tiene perfiles diferentes en ciertos espacios. Por ejemplo, respecto a los problemas de la población en relación con el medio ambiente físico, existe el reconocimiento de que los mismos son resultado, ante todo, de los patrones de desarrollo seguidos por los países agravados por un orden económico internacional profundamente inequitativo. La expresión más grave de los problemas tales como la contaminación ambiental, la erosión y el deterioro de la flora y la fauna se encuentran en los países del llamado tercer mundo, dado que son las condiciones del desarrollo económico y social las que originan los desequilibrios poblacionales y ambientales (CONAPO, 1984c).

En el contex to de México un problema fundamental dentro del binomio población-desarrollo ha sido la conformación y coexistencia en el país de dos situaciones extremas: concentración y dispersión de la población. Tenemos por principio que las tres ciudades más pobladas del país han crecido de forma continua y sostenida en contraste con la generalidad de las demás regiones. Ha sido una constante el crecimiento de las ciudades que han pasado a ser centros de actividades regionales, permaneciendo rezagadas aquéllas cuyos espacios se orientan a actividades agrícolas y de subsistencia. ${ }^{7}$ Los problemas típicos, característicos de la intensa concentración de población (no precisamente problemas generados por ella) son de vivienda en cantidad y calidad mínima: transporte, desempleo o subempleo, delincuencia, falta de seguridad pública, y desde luego deterioro del medio ambiente físico.

Otro ejemplo que muestra la organización territorial de la población en su esquema de concentración-dispersión es la que se forma en el centro

6 Estas consideraciones y otras no menos importantes se encuentran en Roberto Eibenschutz Hartman (CONAPO, 1984b).

En general, se ha manifestado que las aglomeraciones de la población son peculiares en los centros donde son más dinámicos los procesos productivos, observándose en contraste mucho menos población en aquellas regiones de economía menos importante. 
del país, ${ }^{8}$ contando sólo con el $4.4 \%$ del territorio nacional, aloja una tercera parte de la población total (INEGI, 1991a). Frente a los graves problemas que se han gestado junto con la concentración, la política de descentralización formulada desde la década pasada busca, entre otros aspectos, crear una dinámica que tienda a un equilibrio entre población y desarrollo regional; derivado de ello, se ha dado atención a las llamadas ciudades medias para establecer en ellas centros industriales que a su vez permitan el desarrollo de otros centros de población. El propósito de esta política ha operado más bien en ciudades que cuentan con los requisitos de viabilidad de éxito para tal tipo de inversiones y no en las regiones donde verdaderamente atacarían el problema ( Chiapas, Michoacán y Oaxaca, por ejemplo). Tal vez la relación del binomio no se ha hecho explícita en la práctica. Por tanto, se hace necesario un conocimiento de las relaciones que guardan las variables demográficas y la dinámica económica y social por región; de hecho, en este sentido hay que reconocer que se ha realizado un gran esfuerzo. Los trabajos sobre sistemas de ciudades coordinados por el Consejo Nacional de Población ${ }^{9}$ han dado en los últimos seis años nuevos elementos para la política de planeación en materia de desarrollo urbano y regional.

La importancia del enfoque regional al momento de abordar el estudio sobre la población tiene que ver con el reconocimiento de la principal característica del país, su heterogeneidad y disparidad regional. Dentro de su diversidad de regiones, nuestro país cuenta con áreas capaces de absorber población, otras con potencial para retenerla y otras que presentan dificultades para sostener a la que ya tienen. Frente a este hecho, parece ser que parte de la solución a los problemas de la población en nuestro país tiene que ver con el reto que implica generar el desarrollo hacia y dentro de las propias localidades en juego, donde aparte de integrar proyectos nacionales y regionales, se considere la capacidad de recursos e infraestructura. El uso racional de los recursos con los que cuenten las regiones, vinculados a estrategias de desarrollo productivo son, en parte, alternativas posibles. Podemos concluir que el estudio del binomio población-desarrollo debe arrojar datos que permitan una planeación integral y que, sobre

8 Considerando a los estados de Hidalgo, México, Morelos, Puebla, Tlaxcala y el Distrito Federal.

Lo medular de dichos trabajos es que a partir de una visión bastante documentada sobre la distribución de la población en el pás, se manejan sus posibilidades de cambio en los niveles nacional, regional y local. Dichos trabajos ofrecen una caracterización de cada micro-región y de las principales ciudades, según su capacidad para absorber o retener población, de acuerdo con su potencial de desarrollo. Los resultados generales se han centrado en la edición: Sistema de ciudades y distribución espacial de la población en México (CONAPO, 1991b). 
todo, enfatice en la importancia de la población y su entorno social, económico y natural como punto de partida de donde se desprendan las estrategias de desarrollo.

\section{CARACTERÍSTICAS GENERALES DE LA FRONTERA NORTE DE MÉXICO (FNM) ${ }^{10}$}

La región de la FNM ha manifestado procesos particulares de poblamiento y economía que en su conjunto configuran un panorama que se precisa tener presente cuando estudiamos algún espacio de dicha región, digamos por ejemplo el propio proceso de conformación urbana, a fin de lograr un mejor entendimiento de alguna dimensión específica.

Una de las principales características de dicha región es sin duda su acelerado crecimiento poblacional. ${ }^{11}$ Hasta antes de los affos sesenta el crecimiento se ha explicado por los diferenciales en niveles de vida y a la cercanía con un país capitalista desarrollado, vecindad que implícitamente ha implicado una diversidad de oportunidades económicas. Para esa época, otro estímulo al crecimiento tiene que ver con los grandes proyectos de inversión pública dirigidos a alentar la actividad económica de la región a través de la introducçión y mejoramiento de medios de comunicación terrestre. Ya en la década de los sesenta, un factor más de dinamismo y crecimiento para la zona lo ha sido el Programa de Industrialización Fronteriza, que con la industria maquiladora ${ }^{12}$ vino a configurar nuevos procesos, puesto que si en un primer momento el desarrollo de la frontera norte se explicaba por los procesos de migración hacia Estados Unidos, después se asocia en cierta medida con la planta maquiladora, entendida como segmento de la producción mundial e industria globalizada. Es muy importante tomar en cuenta la concentración de la actividad maquiladora en los estados fronterizos, pues para 1991 el $84 \%$ de dichas plantas se ubican en estos estados, manteniendo un personal ocupado de 439,162 personas, lo

\footnotetext{
${ }^{10}$ La FNM la componen seis entidades colindantes con Estados Unidos (E.U.): Baja Califormia, Sonora, Chihuahua, Coahuila, Nuevo León y Tamaulipas. Los municipios estrictamente fronterizos con E.U. suman 38.

${ }^{11} \mathrm{La}$ tasa de crecimiento poblacional de esta zona fue por décadas superior a la presentada a nivel nacional, mientras que en 1940 la FNM contaba con 2.6 millones de personas, para 1990 tenía alrededor de 13.2 millones. Respecto al total nacional pasa de $13.2 \%$ en 1940 al $16.3 \%$ en 1990 . Un panorama general sobre poblamiento en la frontera norte se puede consultar en Gabriel Estrella V., 1988 y Rodolfo Corona, 1991.

12 En este programa se posibilito el libre tránsito en las importaciones de los insumos de la maquiladora, así como la exportación de sus productos libre de impuestos, (excepto por el valor agregado). Esta política directamente abarató los costos de producción de la industria, que aunado a los bajos salarios pagados, estimulo el establecimiento de este tipo de unidades productivas.
} 
cual representa el 89 por ciento de todos los empleos que esta industria ofrece a nivel nacional (INEGI, 1992).

Si bien, el aumento acelerado de la población se ha presentado en toda la FNM, hay que destacar que este aumento ha mostrado un mayor dinamismo en determinados municipios de las entidades fronterizas, ${ }^{13}$ más aún, esta concentración es particular sólo a algunas ciudades; de las fronterizas se cuentan especialmente Ciudad Juárez en Chihuahua, Tijuana y Mexicali en Baja California; y Matamoros, Reynosa y Nuevo Laredo en Tamaulipas. ${ }^{14}$

A pesar de que el crecimiento económico de las ciudades fronterizas ha tenido un comportamiento dinámico y diverso en cuanto a oportunidades de tipo laboral, ${ }^{15}$ el déficit acumulado en infraestructura es ya de considerable magnitud. Los problemas de transporte, comunicación y energía eléctrica, agudizados sobre todo en las principales concentraciones, obstaculizan el óptimo desarrollo de las actividades productivas en general. Por otra parte, la presión sobre la vivienda y servicios públicos (agua entubada, drenaje, energía eléctrica, alcantarillado, vialidades, cobertura en servicios de salud, educación, etc.) tienen una repercusión directa sobre la calidad de vida y bienestar de la población. Hasta el momento, los problemas urbanos identificados en ciertas ciudades fronterizas han rebasado con mucho las políticas y programas de desarrollo en cualquier nivel. A pesar de que la inversión federal para la FNM ha sido muy importante en cuanto a volumen, ésta apenas se orienta a cubrir ciertos rezagos. Así, a pesar de relativos altos niveles de vida en la frontera, del predominio de actividades integradas al mercado y atención de la zona en cuanto a inversión pública y en algunos rubros también privada, el problema común —en algunos espacios más agudo que en otros - es la deficiente cobertura de equipamiento urbano (Guillén, 1990; Fuentes Flores, 1992).

Resulta pertinente señalar que a partir de los trabajos sobre sistemas de ciudades se han identificado 19 ciudades importantes a nivel nacional en donde se recomienda limitar las obras de infraestructura económica intensivas en mano de obra, para evitar que se saturen y se conviertan en zonas

13 Actualmente se concentra en los municipios fronterizos alrededor de 4.35 millones de habitantes, en otras palabras, el $30 \%$ de la población de las seis entidades fronterizas se concentran en una franja de 38 municipios.

15 De las no fronterizas se cuents a Monterrey. Hermosillo y Chihuahua.

15 Como indicadores tenemos el PRB que ha sido más alto que el promedio nacional, la favorable captación bancaria de esta zona y generalmente los bajos indices de desempleo a través del tiempo (Comisión Mexicana de Bancos, 1991: Dirección General de Política Presupuestal, México 1991, en Gonzílez y Corona, 1991). 
con problemas sociales y ambientales (González y Corona, 1991:8); dentro de esas 19 ciudades están consideradas Tijuana, Ensenada, Ciudad Juárez, Matamoros y Nuevo Laredo, entre otras. Por una parte vemos que actualmente se cuenta con el conocimiento y reconocimiento de la problemática urbana de importantes ciudades fronterizas; pero, por otro lado, la definición de la política a seguir no puede resultar tan simple, sobre todo si consideramos las expectativas de crecimiento para la zona. Más que pensar que la FNM y algunas ciudades en particular pueden disminuir la dinámica de crecimiento poblacional y económico, se debe reconocer que en la práctica dicha región sigue apareciendo atractiva para muchos (migrantes, grupos de inversión, etcétera) un atractivo. De hecho, ante las políticas de globalización económica y el Tratado de Libre Comercio (TLC) se ha tendido a creer que en la zona fronteriza más que contraer su base industrial, la extenderá a la provisión de servicios correlacionados con las exportaciones y el mayor intercambio comercial (Alba, 1991:181). En sí, al parecer, cualquier política de desarrollo para la zona debe entonces partir no sólo de la visión integral de los problemas ya existentes e identificados, sino de las expectativas de crecimiento de la zona en sentido amplio. ${ }^{16}$

\section{EXPANSIÓN URBANA Y MEDIO AMBIENTE EN LA CIUDAD DE TIJUANA}

\section{Ciudad y conflicto ambiental}

La ciudad, tema de estudio de innumerables investigaciones, ha sido considerada como un objeto de análisis de primordial importancia ante la aceleración de los procesos de urbanización, sobre todo en los países en vías de desarrollo. Conceptualmente se considera que la ciudad corresponde a un espacio donde interactúan y se configuran relaciones de producción, socialización y de cultura de grupos de individuos. A pesar de ello, pocos la entienden como el medio socioespacial en el cual se asientan actividades y se aglomeran elementos que la constituyen, reproduciendo la vida del hombre y las relaciones sociales de producción; proceso que requiere cada vez más del consumo e intercambio de recursos naturales que al ser utilizados o alterados, repercuten de manera recurrente en el medio ambiente (Schteingart, 1987).

16 Unicamente como ejemplo, es importante observar no solo el comportamiento de la migración interna a las grandes ciudades fronterizas, sino también los flujos de población que desde localidades menos dinámicas de los propios municipios fronterizos y aun de áreas rurales de los mismos, se trasladan a sus centros más importantes. 
La expresión más notoria de la degradación ambiental se encuentra, por lo tanto, en las ciudades, aunque día a día su magnitud y expansión abarca espacios rurales y muy remotos. Por tanto, se hace patente la necesidad de integrar el factor ambiental en los planteamientos políticos y académicos como parte de la problemática que, tanto a nivel local, regional y nacional, incide activamente en la calidad de vida de los habitantes urbanos y en la configuración espacial de los asentamientos. La calidad de vida poblacional está directamente relacionada con los grados de satisfacción de las necesidades tanto objetivas como subjetivas del ser humano, donde el estado ambiental, junto con la capacidad de la organización social para satisfacer esas necesidades, influye en los niveles de calidad en función de juicios de valor establecidos por la sociedad, tomando en cuenta los requerimientos para conservar la salud y los patrones y valores sociales y culturales (Gallopín, 1986).

El crecimiento de las ciudades como parte del proceso poblacióndesarrollo ha traído consigo una serie de fenómenos problemáticos que van desde una desigualdad social, evidente en la diferenciación de la ocupación del espacio-territorio, expresado en los diferentes niveles de consumo colectivo, hasta el deterioro de los niveles de calidad de vida. Estos problemas se manifiestan en la degradación del medio físico que ocasiona contaminación de recursos, desforestación, desabasto de agua y áreas verdes, dificultad en la eliminación de desechos sólidos, ocupación irregular de las zonas inaccesibles para la urbanización, etcétera. Sin embargo, pese a esos altos ritmos de crecimiento, los problemas ambientales de las zonas urbanas no pueden explicarse sólo en términos de la magnitud poblacional o espacial, sino tomando en cuenta las relaciones que cada asentamiento concreto establece con sus recursos a través de las múltiples y variables mediaciones tecnológicas que intervienen en la producción de bienes y servicios.

Este apartado se centra precisamente en el efecto del desequilibrio ecológico en una ciudad en términos del desbalance entre el crecimiento urbano y la capacidad de la ciudad para sustentar un desarrollo equilibrado respecto a la disponibilidad de recursos y, a los efectos contaminantes y perturbadores del espacio. Se escogió a la ciudad de Tijuana por ser un centro urbano representativo de contradicciones entre su dinámica económica y su proceso de urbanización. Se hace una caracterización del crecimiento poblacional y expansión física de la ciudad en relación con su disponibilidad de recursos en infraestructura y sus consecuencias en los niveles de calidad de vida de la población, desde el punto de vista de una alteración ambiental en la que se involucran procesos de contaminación y desmedida ocupación del territorio. 


\section{Proceso de conformación urbana en Tijuana}

El proceso de urbanización que muestra la ciudad de Tijuana es representativo del fenómeno en las ciudades de la frontera, con características comunes en cl acelerado crecimiento de la población con alto ingrediente migratorio. Por otro lado, la expansión territorial es el reflejo de una serie de factores motivados por su colindancia con el estado de California, E.U., lo cual le confiere una dinámica económica y demográfica de formas y procesos particulares.

El crecimiento de la mancha urbana de la ciudad presenta una evolución bastante influenciada por los acontecimientos fronterizos caracterizados geopolíticamente. Para 1900, el poblado contaba tan sólo con 242 habitantes y 30 años después se incrementan a 11,677. Este importante aumento de población se entiende dentro del contexto de la situación moralista prevaleciente en Estados Unidos que promulga la ley seca, con la cual se intensifica el paso de turistas a Tijuana, aspecto que a su vez motiva el primer gran impulso económico de la ciudad.

Se considera que la ciudad inicia su desarrollo espacialmente de manera concéntrica, alrededor del centro tradicional, en dirección a las áreas contiguas de fácil adecuación para el uso urbano. En la década de los veinte la ciudad se dinamiza aumentando la ocupación de suelos aptos, proceso que más tarde, con la crisis mundial de 1929, disminuye sólo coyunturalmente. El régimen de zona libre, instaurado más tarde, favorece las relaciones comerciales y fomenta una estructura económica de servicios que sigue alentando el crecimiento de la población y en consecuencia de la ocupación territorial. La tasa de incremento poblacional entre 19301940 fue de $7.14 \%$ y en la siguiente década llega a la más alta registrada, es decir, del 13.42. Gran influencia tuvo la situación propiciada por la segunda guerra mundial, tanto por el paso de militares de Estados Unidos a México, quienes se introducían temporalmente con fines de distracción, como por la migración proveniente del interior del país llamada a ocupar puestos agrícolas en tierras norteamericanas, respondiendo al programa de braceros. En el periodo 1950-1960 se muestra la más rápida expansión urbana que se inicia con la ocupación de terrenos no aptos y condicionados (entre 15 y 30 y más de $30 \%$ de pendiente). Para $1940 \mathrm{el} \mathrm{fundo} \mathrm{legal} \mathrm{de} \mathrm{la}$ ciudad era alrededor de 836 hectáreas que a la postre serían insuficientes, puesto que la expansión urbana determinada por un crecimiento poblacional acelerado, rebasaría con mucho los intentos por delimitar esa área (Hiernaux, 1986:59).

Posteriormente, la deportación masiva de los "wetback" (mexicanos con residencia ilegal en Estados Unidos), repatrió a un gran número de 
mexicanos trabajadores agrícolas después de la guerra de Corea (1953), situación que propició la proliferación de asentamientos en suelos no aptos y condicionados, logrando un perímetro de aproximadamente 5 kilometros a partir del centro. En este periodo se inicia el crecimiento hacia el este del centro en predios ex agricolas de La Mesa, dando inicio a una expansión longitudinal a lo largo de la carretera Tijuana-Mexicali (Hiernaux, 1986). Como resultado del programa maquilador en la década de los sesenta (1965) se crea la necesidad de incrementar la infraestructura en la ciudad, propiciando con ello mayor estímulo al crecimiento poblacional. De 165,690 habitantes en 1960, la ciudad pasa a contar con 461,257 en 1980. Se da inicio a los primeros fraccionamientos legales e ilegales periféricos, que acentúan las necesidades de infraestructura social en la ciudad. Los problemas de abasto de servicios públicos como el agua potable, se hacen patentes, y la disputa por el suelo y vivienda se ve enmarcada en un contexto de desigualdad socioeconómica, dando como resultado una disparidad en la distribución del espacio urbano (Hiernaux, 1986). En esta década de 1970 a 1980, el crecimiento desordenado se ubica sobre terrenos inaccesibles, la mancha urbana cubre 6,500 hectáreas de las cuales ya el $16 \%$ es ocupada sobre terrenos inapropiados (SAHOPE, 1984). No obstante los esfuerzos gubernamentales por ordenar y regular el crecimiento de la ciudad, se agudizan los conflictos en los usos del suclo por el incremento y diversificación de actividades como la industrial. Los problemas implicitos a la aglomeración urbana dados en este espacio son, entre dtros, desabasto de servicios en áreas irregulares o inaccesibles, especulación del suelo, encarecimiento de la vivienda y, sobre todo, deterioro de las condiciones del medio físico que empieza a manifestarse alterando los niveles de calidad de vida de la población. Desde 1970, se intensifica la ocupación de las áreas no aptas y condicionadas en los cañones y barrancas al suroeste de la ciudad. El crecimiento de la mancha se caracteriza, entonces, por su seguimiento hacia las principales vías de comunicación interurbanas, en dirección de Tecate principalmente, en una ocupación urbana de no más de 500 metros a ambos lados de la carretera (Ranfla, et al., 1989:322).

Según datos censales, en 1990 el municipio de Tijuana registró 747,381 residentes habituales asentados sobre una superficie de 14,184 hectáreas (área neta ocupada). El 33\% del área está ocupada por barrancos, cañones, cerros y lomeríos. Además de los grandes baldíos que representan áreas potencialmente urbanizables que, al no ser ocupadas, producen problemas en la dispersión de redes de infraestructura. El crecimiento de los últimos años se ha dado en zonas inaccesibles, entre 1985 y 1991 la mancha se expandió 2,336 hectáreas, de ellas más del $50 \%$ fue sobre cerros y cafiones ubicados al sur de la ciudad (ver figura 1), área considerada como de 
"preservación ecológica", que al ser ocupada irregularmente provocan escenarios de alteración al medio ambiente (SAHOPE, 1991) y conflictos entre grupos de interes.

\section{Alteraciones ambientales por cambio en el uso del suelo}

Cuando se habla de degradación de los suelos no se puede dejar de lado el fenómeno de expansión de la mancha urbana, donde la disminución de áreas cultivadas promueve cambios de la estructura productiva y altera el equilibrio ecológico. Para la ciudad de Tijuana este esquema se dio en poca proporción, ya que su base económica ha estado orientada al comercio y servicios, actualmente combinada con actividades industriales.

Como se observa en el apartado anterior, la expansión físico-demográfica de la ciudad, está enmarcada por periodos de intenso incremento mantenido hasta los últimos años. La actual configuración de la ciudad se ha enfrentado al reto de desarrollarse en un ambiente natural no del todo propicio para los asentamientos urbanos, específicamente por su perfil topográfico, factor que ha influido en gran medida en el impacto que esa expansión causa al medio ambiente, tomando en cuenta que la ciudad se encuentra asentada en gran parte sobre el valle del rio Tijuana, limitando al oeste y suroeste con zonas de cerros y lomeríos que constituyen una barrera natural entre la ciudad y el oceáno Paćfico; al oriente, con el cerro del Colorado y la Mesa de Otay. Según el rango de pendiente, el $56.5 \%$ del área urbana estudiada en el Plan de Desarrollo Urbano de Tijuana (PDUT), corresponde a terrenos que por su escasa o poca pendiente (de $0 \%$ a $15 \%$ ) no representa mayor problema para la edificación, infraestructura e introducción de servicios como drenaje y agua potable. El 19.5 del área territorial corresponde a terrenos con pendiente moderada (de 15 a 30\%), pero que ya representan problemas para su urbanización. El resto (24\%) está dentro del rango de pendiente de más de $30 \%$, que se consideran inadecuadas para la urbanización, debido a los altos costos de instalación y el mantenimiento de la infraestructura (SAHOPE, 1991).

Independientemente de las inversiones que por parte de autoridades y de la población misma se tienen que efectuar en obras de infraestructura, adecuación y prestación de servicios, el problema de la expansión de los asentamientos humanos sobre terrenos topográficamente inadecuados propicia una serie de procesos que van en detrimento del medio ambiente circundante, situación que propicia problemas como:

La ocupación en pequeños valles y laderas abruptas que contribuyen a la desaparición de la cubierta vegetal natural (matorrales y pocos pastizales) que representan una protección al suelo en contra de la erosión hídrica y eólica. 
E. $\mathbf{u}$.

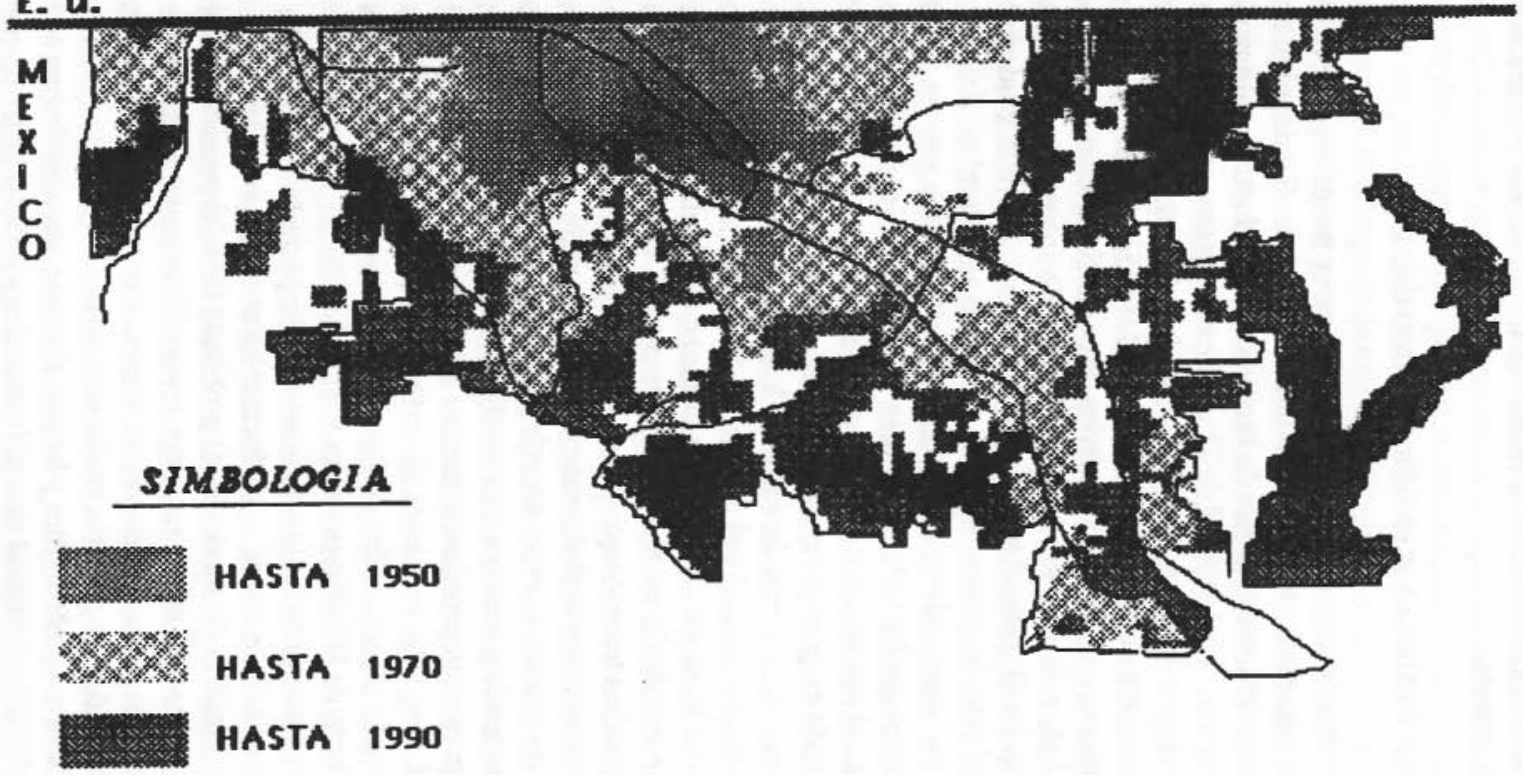

Figura 1. Crecimiento histórico de Tijuana 1950-1990.

Fuente: Ranfla y Álvarez (1986), "Expansión física, formas urbanas y migración en el desarrollo urbano de Tijuana 1900-1984". La información actualizada hasta 1990 fue elaborada por las autoras. 
en donde en esta última el viento es agente importante de contaminación por partículas sueltas de polvo y otros ingredientes.

A falta de vegetación contenedora, los escurrimientos superficiales se intensifican arrastrando grandes cantidades de suelo de las partes altas a las bajas, contribuyendo a la saturación de vialidades y canales. Además de que por el acelerado escurrimiento, la infiltración se ve afectada y con ella la alimentación de los mantos freáticos.

Finalmente, estos asentamientos periféricos sin acceso a servicios públicos, independientemente de su ubicación en zonas aptas o no aptas, contribuyen, en gran medida, a la contaminación de los mantos freáticos por desalojo de aguas residuales vía fosas sépticas; además, por la práctica de incineración de tiraderos de desechos sólidos a cielo abierto, perjudicando con ello la salud de los habitantes por las emisiones de gases tóxicos.

En suma, los problemas derivados de una inadecuada ocupación del suelo urbano, han contribuido a la conformación de una ciudad desadaptada con su medio ambiente. Por un lado, la distribución de áreas habitacionales carentes de servicios públicos en torno a centros industriales ha propiciado problemas de desechos tóxicos, especulación de grandes terrenos, baldíos que incrementan los tiraderos de desperdicios y en sí contaminación. Por otra parte, la degradación del suelo debida a la ocupación de terrenos no aptos de preservación ecológica, además de alterar el equilibrio natural, representan problemas que dañan los niveles de calidad de vida de la población.

En el proceso de desarrollo urbano de ciudades como las de la frontera norte de México, como es el caso de Tijuana, los problemas derivados de las desigualdades entre los ritmos de crecimiento económico y demográfico, ha dificultado cubrir en la cantidad y calidad necesaria los servicios colectivos, vivienda e infraestructura, así como mecanismos de protección ambiental adecuados. Esto explica en parte por qué se ha llegado en las últimas décadas a determinados niveles de degradación ambiental, evidente en la expansión desordenada de la mancha urbana, donde la población se ve expuesta al deterioro de sus niveles de salud y bienestar. Gran parte de los recursos naturales se ven afectados (agua, vegetación nativa, suelo, aire) impactando las relaciones entre los elementos naturales a través de la disminución de zonas de reserva de áreas verdes, la acelerada erosión del suelo, alteración de mantos freáticos y contaminación del aire.

Enseguida se destacan algunos de los problemas más patentes relacionados con la degradación del medio ambiente y su efecto en el bienestar de la población. La información disponible es escasa y desintegrada, por lo que muy probablemente el problema está subestimado hasta el momento. 
Sin embargo, la semblanza general es de utilidad para poder plantear estudios más concretos sobre un espacio y población definida.

\section{Contaminación por aguas residuales}

Conviene señalar que la conformación de la ciudad de Tijuana se ha dado en importante medida bajo la influencia de su contigüidad espacial de naturaleza binacional con San Diego, una de las ciudades más importante de California, E.U. que también ha crecido aceleradamente y que en cuanto a población es 2.5 veces más grande que Tijuana. La conurbación de estos dos importantes asentamientos ha generado procesos trasnacionales en distintos rubros; uno de ellos tiene que ver con el medio ambiente que comparten dichas ciudades.

Si consideramos que los fenómenos ambientales no respetan fronteras ni ideologías, es posible afirmar que el problema de la contaminación del agua en la región corresponde a un conflicto binacional, se ha destacado que tanto el crecimiento de Tijuana como el de San Diego, representan uno de los núcleos urbanos más dinámicos de la región, por lo que la expansión urbana ha vuelto más complejo el manejo de su sistema de drenaje, alterando recíprocamente la composición del agua en sus costas y muy probablemente en los depósitos subterráneos, producto del contacto con las aguas servidas (de desecho) de ambas ciudades (Sánchez, 1989).

La expansión incontrolada de los asentamientos humanos agotó rápidamente la escasa planicie generada por el cauce del río que atravieza la ciudad de Tijuana, provocando con ello la ocupación irregular o legal de terrenos en áreas no adecuadas para la urbanización, lo que llevó a que los sistemas de drenaje y agua potable representaran altos costos que la mayoría de la población no puede solventar. El problema en el lado mexicano se origina por su derrame en tres parte de la frontera: a) el río Tijuana, b) a lo largo de la avenida Internacional entre la ciudad y Playas de Tijuana (asentamiento ubicado en el extremo noroeste de la mancha urbana), básicamente en la zona de los cafiones que desembocan en la frontera internacional y c) las descargas en el litoral en Playas de Tijuana. El flujo que se vierte hacia el río sigue el drenaje natural de la zona, cruzando la frontera y desembocando en río Tijuana y playa norteamericana (Sánchez, R. 1988:20). Pese a los acuerdos y esfuerzos realizados con inversiones de instituciones internacionales y nacionales, como la Secretaría de Desarrollo Urbano y Ecología (SEDUE), (actualmente Secretaría de Desarrollo Social, (SEDESOL) para construir estaciones de bombeo y plantas de tratamiento, ${ }^{19}$ la solución para el problema de drenaje no ha sido la mejor. Técnicamente hablando el flujo centralizado de 
drenaje tiene que subir 100 metros para llegar a la costa para su tratamiento, haciendo riesgosa su transportación.

La ciudad continúa teniendo el problema de descontrol del sistema de aguas servidas a través del río Tijuana. Según la Comisión Internacional de Límites y Aguas (CILA) este flujo de aguas es actualmente superior a los 12 millones de galones por día, manteniendo e incrementando los niveles de contaminación en las playas y en el área del estuario del río Tijuana. De acuerdo con un reporte preliminar, se establece que en la frontera de México-Estados Unidos y San Antonio del Mar, Baja California, se detectaron niveles de contaminantes por residuos coliformes fecales al nivel o muy por encima de los recomendados para zonas de recreación (200 fecales $/ 100 \mathrm{ml}$ ). Detectando además un descenso considerable en el número de especies de algas marinas (Morales, 1986:224-225). La ciudad de San Diego también presenta graves problemas en su manejo de aguas servidas, pues la ciudad cuenta con un sistema centralizado que concentra en una planta de tratamiento el $90 \%$ de efluente de todo el condado, localizada en Point Loma, hacia el extremo noroeste del centro de la ciudad de san Diego. El crecimiento de la ciudad ha rebasado los esfuerzos para mejorar el sistema de drenaje, observándose fallas de derrame en diversos puntos de la costa.

Existe una importante relación entre el conflicto ambiental con el comportamiento de las corrientes marinas, ya que éstas afectan tanto del lado americano como del mexicano -aunque según estudios realizados recientemente, las corrientes tienden a afectar más al recorrer en dirección norte a sur, en mar adentro, pues transporta los desechos de la planta de Point Loma en San Diego, hacia aguas mexicanas-; en tanto las corrientes superficiales siguen una dirección sur a norte, cercanas a la costa, donde se afecta la zona de Imperial Beach (lugar localizado hacia el suroeste del Condado de San Diego, frente a las costas de Playas de Tijuana y San Antonio del mar, del lado mexicano) por los desalojos del río Tijuana. Con esto se muestra que el conflicto referente al problema de contaminación en la región obedece de manera importante al proceso de desarrollo que ambas ciudades han experimentado, cada cual con sus ritmos y niveles correspondientes. Mientras no se acepte la responsabilidad en cada caso y se articulen medios de solución comunes, el problema llegará a dimensiones incontrolables, especialmente cuando en tratados recientes se anuncia una gran inversión industrial en la cual deben ir programas paralelos de

19 La SRDUB construy6 una nueva estación de bombeo en 1986, en 1987 construy la planta de tratamiento primario avanzado en San Antonio del Mar, localidad al noroeste de la ciudad, 
inversión que contrarresten el déficit que en infraestructura social se tienen y que provocan la contaminación local y regional.

\section{Contaminación del aire}

Al igual que con el conflicto binacional propiciado por la contaminación del agua en la costa del Pacífico, existe el problema de la contaminación atmosférica en la cuenca Tijuana-San Diego, donde ambas ciudades comparten el mismo espacio atmosférico, con un intercambio diario de masas de aire y por lo tanto de contaminantes. Por las características atmosféricas, la región está dominada por un sistema de alta presión localizado en el océano Pacífico, el cual mantiene un flujo de vientos del noroeste hacia tierra adentro. Las diferencias de temperaturas entre mar y tierra dan origen a un ciclo de vientos locales durante el día, volviéndose sistemático de acuerdo con las estaciones del affo, contrastando en verano. La cuenca tiene un patrón cíclico de vientos ligeros por la mañana que transportan las emisiones matutinas de las paredes del cañón del río Tijuana hacia el océano; horas después el flujo de vientos se revierte llevando la contaminación de mar a tierra. En otras condiciones meteorológicas, las masas de aire del sur de Los Ángeles, California, E.U. pueden ser transportadas a la cuenca, provocando un incremento en los niveles de contaminación que inciden de abril a septiembre. Se ve afectada también la porción mexicana, pero debido a la carencia de un sistema de monitoreo adecuado, se desconoce la magnitud del problema. La principal fuente de contaminación en el aire ha sido la termoeléctrica de la CFE, de Rosarito, Baja California, con emisiones de bióxido de azufre (S02) y bióxido de nitrógeno (NO2), asimismo la cantidad de autos circulantes en la ciudad, aproximadamente 130 mil vehículos de los cuales más de un $85 \%$ carece del sistema para el control de las emisiones que son responsables de la mayor parte del monóxido de carbono y nitrógeno (Álvarez, 1988:88).

Por otro lado, la segunda fuente fija de contaminación la constituye la quema de basura, que con la implementación del relleno sanitario por parte de SEDUE y el municipio se ha reducido en gran medida, aunque existen aún fuentes importantes como las ladrilleras ubicadas en la Mesa de Otay, las que a finales de 1970 utilizaban como material combustible desperdicios de llantas y otros productos de hule, que generan fuertes emisiones de bióxido de azufre (Álvarez, 1988).

Según el análisis realizado por la SEDUE, el diagnóstico de la calidad del aire en la ciudad se realizó a través de tres estaciones de monitoreo ubicadas en: a) Casa del Voceador, en el centro de la ciudad en una zona comercial de alto tránsito; b) La Mesa, localizada al este de la ciudad en 
una zona habitacional, comercial, e industrial y c) La Presa Abelardo L. Rodríguez, al sureste de la ciudad en una zona habitacional cercana a la carretera libre Tijuana-Tecate, contigua a la mancha urbana. En dos de las tres estaciones de monitoreo de la ciudad, la calidad del aire de acuerdo con el índice metropolitano de calidad del aire (IMECA) es satisfactoria o buena durante la mayor parte del año. Sin embargo, en la estación de La Mesa el criterio se rebasa por lo menos una vez al mes. Los vientos procedentes del noroeste acarrean contaminantes hacia la Presa Abelardo L. Rodríguez, donde la topografía de la zona constituye una barrera natural que impide su difusión, por lo que en ocasiones la calidad de aire de la zona se califica como no satisfactoria (SEDUE, 1986:43).

\section{Contaminación por desechos sólidos}

El término de desecho sólido urbano (al que comúnmente se denomina basura) se utiliza frecuentemente para designar a los desechos que se producen en una ciudad como consecuencia de las diferentes actividades domésticas, comerciales y de servicios, excluyendo a los de hospitales e industria debido al alto riesgo en su manejo, que requieren de unidades de tratamiento distinto.

La magnitud del problema de recolección y disposición en las concentraciones urbanas representa una tasa de generación en muchos casos mayor que el promedio nacional, del orden de 0.7 a $1.0 \mathrm{Kg}$. Whab. Uía y la densidad es ligeramente menor, del orden de 0.3 a 0.4 ton $\backslash$ metro cuadrado; esto en ciudades medianas (de 300 mil a 500 mil hab.) representa una generación de basura de alrededor de 250 toneladas por día (Trejo, 1987:80-81).

En casi todas las ciudades es común depositar la basura en sitios no muy lejanos de la urbe, sin someterla a ningún tratamiento. Se ha considerado que los factores que intervienen en esta situación son los siguientes: ignorancia sobre el riesgo para la salud; desconocimiento sobre las técnicas de tratamiento; falta de planeación en el crecimiento de las ciudades; escasos recursos económicos para atender el servicio; una actitud combinada entre apatía del ciudadano y actitud irresponsable de autoridades y existencia de fuertes intereses económicos particulares que se benefician con la recuperación de materiales de desecho. ${ }^{20}$

\footnotetext{
${ }^{20}$ Miles de llantas cuya existencia representan daños a la comunidad, en cuanto al latente riesgo por su material inflamable, permanecen en todos los sitios de la ciudad de Tijuana debido a la falta de un confinamiento adecuado. En la delegación local de SEDESOL se tienen registrados 131 establecimientos que se dedican a la venta de llantas usadas; $\mathbf{y}$ aunque en la actualidad está suspendida la importación, algunos negocios aún no agotan sus permisos y continúan importando esos materiales (Argelia, 1992).
} 
En Tijuana, la disposición de los desechos sólidos es uno de los problemas más serios, en especial por su topografía que ha obligado a gran parte de la población a ocupar cañones, cerros y lomeríos a los que el servicio de recolección de desechos no tiene acceso, convirtiendo espacios como barrancas, baldíos y cañadas en tiraderos en los que sobresalen productos no biodegradables como envases y envolturas de material sintético. Se ha calculado que en la ciudad se generan aproximadamente 850 toneladas de basura al día, el municipio cubre sólo la recolección de una tercera parte, que aunado con el servicio que ofrece una empresa privada, se incrementa a un 50\% (Álvarez, 1988).

Las consecuencias lógicamente son muy negativas, la proliferación de tiraderos a cielo abierto ocasionan, entre otros, problemas de reproducción de insectos domésticos; proliferación de roedores; malos olores; gérmenes patógenos; incendios y contaminación del aire, agua, suelo y degradación del área en general. ${ }^{21}$

\section{Contaminación por industria}

El auge industrial que trajo consigo el impulso a las maquiladoras en las ciudades de la frontera ha repercutido constantemente en el medio ambiente y en la salud mediante procesos de contaminación, en especial en cuerpos de agua superficial y subterránea. Existen muchos casos donde se han encontrado residuos de contaminantes industriales en las aguas negras municipales, como en Tijuana y Mexicali. ${ }^{22}$

El deterioro se debe principalmente a la generación de desechos tóxicos y peligrosos, así como a manejo de materiales químicos de alto riesgo. Pese al gran interés por los temas ambientales observado en el proceso de negociación del Tratado de Libre Comercio, ha quedado explícita la limitación de las instituciones gubernamentales mexicanas para controlar la vigilancia sobre la maquila, $y$ en general sobre los problemas ambientales. "Según información de SEDUE, de las 1963 plantas establecidas en México hasta fines de 1990, cuando menos 1,036 son consideradas importantes generadoras de residuos peligrosos. De ellas, sólo 33.5\% cumplen con los requisitos de la legislación ambiental mexicana" (Sánchez, 1991:9). Aunque el dato está referido a nivel nacional, resulta importante

${ }^{21}$ Habitantes de colonias populares se quejan de la acumulación de basura que es quemada al aire libre en lugares inapropiados. De hecho han señalado que en mucho tiempo (años) no han contado con un servicio normal de recolección. El desagüe corre a cielo abierto llevándose los desperdicios depositándolos a más de dos kilómetros, tapando las alcantarillas, lo que produce mayores problemas para la ciudad (Rentería, 1992).

22 Para mayor información ver Sánchez, 1991. 
bajo la consideración de que la actividad maquiladora se ha concentrado en ciertas ciudades fronterizas y entre ellas, especialmente Tijuana.

La creación del Plan Integral Ambiental Fronterizo (PIAF) ${ }^{23}$ trajo una nueva panorámica en el grado de interés de los conflictos ambientales en la frontera, aunque este esfuerzo en sí mismo no es suficiente si no se aplican los recursos necesarios para llevar a cabo el plan, si permite el inicio de negociaciones que evidentemente tendrán repercusiones positivas en la aplicación de normas ambientales que contribuyan al control de la contaminación industrial. Sin subestimar el problema, los riesgos ambientales aún son altos, en especial para aquellas áreas residenciales contiguas a zonas industriales donde los desechos de algunas plantas son vertidos directamente al drenaje público o al suelo directamente. ${ }^{24}$ Lo anterior, da un indicio de una situación que va a repercutir en la generación de desechos tóxicos industriales que afectan de manera muy importante al medio ambiente y trae consigo graves consecuencias a la población, sobre todo a la más vulnerable dada su ubicación espacial.

Se tiene conocimiento de un estudio realizado en el poblado La Gloria, al sur de Tijuana (Álvarez, 1988), donde se constató que actividades industriales manejan una considerable cantidad de substancias tóxicas y peligrosas como insumos intermedios o auxiliares en la producción, por ejemplo, solventes, metales, ácidos, fibra de vidrio, cromo, etcétera. El objetivo del estudio era localizar la fuente principal de contaminación en la región, estableciendo correlación entre niveles de contaminación y distancia a la fuente. $\mathrm{Al}$ respecto se encontró contaminación por plomo provocado por una recuperadora de este metal a partir de baterías usadas (Pérez y Reyes, 1986 en Álvarez, 1988:88).

\footnotetext{
${ }^{23}$ Este plan representa la primera etapa de un programa binacional de protección ambiental froteriza y se preparó conjuntamente por la SEDUB y la Agencia de Protección Ambiental (EPA) de los Estados Unidos en 1992.

24 Se ha señalado el fracaso que en los tres niveles de gobierno ha agravado la solución del problema de contaminación industrial generada por industrias ubicadas al lado de zonas marginadas de Tijuana. Residentes de asentamientos localizados en la periferia han solicitado el auxilio de la comisión de Derechos Humanos y de autoridades de San Diego. Se ha denunciado la proliferación de múltiples enfermedades que afronta la población, sobre todo la niñez, afectada por desechos industriales que corren a "cielo abierto", entre otros desperdicios (Martínez, 1992). Por otra parte, se ha dado a conocer la existencia de sitios en la Delegación de la Presa, al sureste de la ciudad, en los que se queman al aire libre materiales de desecho industriales como cobre y plástico PVC, recogidos de algunas industrias de la región y otros atraídos por la garita de Tecate (el municipio de Baja California más próximo a Tijuana en dirección Este), procedente de Estados Unidos. Personal de la Unidad de Protección Civil de Tijuana han señalado que esta situación no es nueva, ya que hace dos años el delegado de $\mathrm{La}$ Presa denunció la incineración de éstos materiales (Lu, 1992).
} 
En general, en este apartado se ha puesto de manifiesto parte de los problemas ambientales que en la ciudad de Tijuana provocan un desequilibrio ecológico, en otras palabras, alteración de los sistemas naturales que se encuentran interrelacionados formando el paisaje del medio ambiente. Como se ha expuesto ya, el desbalance entre el crecimiento urbano y la capacidad de la ciudad para absorber este crecimiento de forma ordenada ha sido y es uno de los problemas fundamentales que la ciudad tiene ahora. En conjunto, la situación descrita repercute sobre los niveles de calidad de vida de la población, que desde esta perspectiva ambiental parece estar muy por debajo de lo requerido y más de lo deseado. Los problemas inherentes a la topografia de la ciudad, los derivados de la industria, del crecimiento económico y poblacional, aunados al intenso uso y demanda de servicios de la población, requieren en conjunto redimensionarse dentro de las políticas de desarrollo y medio ambiente para la zona.

\section{CONSIDERACIONES FINALES}

Resulta actualmente inaceptable desprender de cualquier plan de desarrollo regional, industrial o de cualquier otro tipo, los procesos ecológicos naturales dentro de los cuales vive el hombre. Por tanto, resulta inadmisible caer en una posición de inercia donde parezca justificarse el deterioro ambiental a cambio del desarrollo; finalmente un desarrollo integral sólo puede ser posible en tanto tienda a mantener equilibrio con el medio ambiente.

Una educación ecológica es básica hoy en todos los niveles y en todos los grupos de representación social. Tal educación debe de hacer su primer labor en la creación de conciencia acerca de la relevancia de conservar un medio ambiente favorable a la vida del hombre, $y$ en general de la vida de la tierra. Bajo esta prioridad del tomar conciencia cada cual debe tomar su responsabilidad. Si bien sabemos que aunque todos formamos el ambiente que tenemos, es claro que existen niveles de responsabilidad y por lo tanto de compromisos. En el nivel de espacio geográfico donde centramos este trabajo, la relación de la sociedad frente al problema ecológico se vuelve compleja.

El deterioro del medio ambiente de nuestro entomo más directo es un problema común. Dentro de los propósitos de soluciones ha habido una serie de convenios, programas, propuestas y presupuestos asignados. Todo ello para convenir una solución conjunta del problema. Quizá uno de los mejores resultados de esta comunicación —en importante medida comunicación transfronteriza binacional - no sean las reuniones de representación diplomática de altos y bajos niveles de autoridad de los diferentes 
países para comentar el problema, sino el resultado concreto de proyectos técnico-científicos de investigadores mexicanos y norteamericanos, porque finalmente estos trabajos son los que tienden a conocer a fondo una situación ambiental desde el momento que se generan diagnósticos sustentados a partir de mediciones científicas de diferentes elementos ambientales. Por lo mismo se supone deben de tomarse como instrumento de argumentación en el momento de asignar responsabilidades y compromisos.

Los problemas de concentración, pauperismo, marginación y presión sobre los servicios públicos, entre otros, seguirán agudizándose. Resulta ingenuo pensar que tales problemas se "suavizarán" con medidas de política estatal y de redistribución de la población. Debido a que el proceso de configuración urbana mostrado en la ciudad de Tijuana corresponde a una multiplicidad de factores que en sí mismos mantienen una dinámica propia, fuera de la contemplación de algunas estrategias oficiales de desarrollo, es difícil pensar que parte de la solución sea la posibilidad de que la ciudad deje de crecer. El crecimiento es implícito a esta ciudad, su ordenación y adecuación a requerimientos sociales y ambientales para proporcionar los mejores niveles de bienestar, constituye el gran reto. Por ello, más bien hay que pensar que el crecimiento poblacional y económico se seguirámanifestando y con ello la demanda y presión de los servicios públicos, y - aunque no necesariamente - la afectación del medio ambiente.

Es difícil pensar que, en un nivel agudo del problema, únicamente sean el municipio o el estado los que debieran de enfrentar la situación. Reconociendo desde luego su competencia, se debe pensar en todo el potencial de los otros actores responsables también de solucionar con su participación parte del problema; los grandes grupos privados de inversión, háblese del turismo, el comercio y en importante medida la industria, tienen un papel fundamental en su solución, tanto por su ingerencia en asunto de responsabilidades, como por el mejor desarrollo de sus actividades económicas (comercio y servicios) y productivas (industriales, maquiladoras en particular). Otro sujeto social que se pensaría más interesado en el mejoramiento de su medio ambiente es la población; al respecto, diferentes grupos dentro de la sociedad civil suelen organizarse para enfrentar en conjunto los problemas comunes. En un nivel se han orientado a denunciar y presionar ante la autoridad pública; el otro nivel, el de proponer, orientar y promover una educación ecológica, está emergiendo. 


\section{BIBLIOGRAFÍA}

ALBA, Francisco. 1991. "La creación de un área de libre comercio en América del Norte y sus efectos en la frontera norte de México: elementos para un pronóstico". (Nota crítica) Frontera Norte, vol. 3, no. 6, julio-diciembre.

ÁLVAREZ, Juan. 1988. "El medio ambiente en el desarrollo de la frontera norte de México". Cuadernos de economía, serie 3, no. 5, UABC, México.

BARRADAS, V, y J. Seres R. 1988. "Los pulmones urbanos". Ciencia y Desarrollo, enero-febrero, CONACYT.

CANTÚ, Anibal. 1990. Apuntes sobre planeación para alumnos de posgrado en Desarrollo Regional, COLEF, generación 1988-1990.

CONAPO. 1984a. Población, recursos, medio ambiente y desarrollo. México.

- $\quad$ 1984b. Conferencia Internacional de Población en México. México.

- $\quad 1984 \mathrm{c}$. La población y el desarrollo regional y urbano. México.

- 1988. Demografía de la Frontera Norte de México. México.

- 1991a. Programa Nacional de Población.

- 1991b. Sistema de ciudades y distribución espacial de la población en México. Tomos I y II. México.

CORONA, Rodolfo. 1991. "Principales características demográficas de la zona fronteriza del norte de México." Frontera Norte, vol. 3, no. 5, enero-junio. COLEF.

CUTLER, S. Laurence y Sherrie S. Cutler. 1992. Reclycling Cities for People. The Urban Desing Process, Boston, CBI, Publishing, Company, Inc., 2a. ed.

EIBENSCHUTZ, Hartman R., Eduardo Juárez Aguirre y Napoleón Miranda Melo. 1984. "La Población en la Planeación de los Asentamientos Humanos". La Población y el desarrollo regional y urbano, CONAPO, México.

ESTRELLA V., Gabriel. 1988. "Migraciones interurbanas en la frontera de México". Ponencia presentada en la Reunión Anual de Population Association of America, Nueva Orleans, IIS-UABC.

FUENTES F., César. 1992. "Proceso de Industrialización en ciudad Juárez: experiencias y alternativas en el financiamiento de la productividad urbana". (Mimeo), CoLEF, Ciudad Juárez, Chihuahua.

FUENTES, David. 1984. Desarrollo urbano y fuerza de trabajo en la frontera norte de México, el caso de Tijuana. (Reporte terminal de investigación). Instituto de Investigaciones Sociales de la UABC, Mexicali, Baja California, México. 
GALLOPÍN, Gilberto. 1980. "Ecología y Desarrollo: evolución y perspectiva del pensamiento ecológico" O. Sunkel y N. Gligo (compiladores), Estilos de Desarrollo y Medio Ambiente en América Latina, México, FCE, vol. 1.

- $\quad$ 1986. "El medio ambiente humano." Enrique Left. Los Problemas del conocimiento y la perspectiva ambiental del desarrollo. Edit. S. XXI, México.

GONZÁLEZ García de Alba, Ligia y Reina Corona. 1991. "Distribución de población, migración y desarrollo regional en la frontera norte". Coloquio sobre política de población en la frontera norte de México, 3 y 4 de octubre.

GUILLÉN Tonatiuh. 1990. "Servicios públicos y marginalidad social en la frontera norte". Frontera Norte, vol. 2, no. 4, junio-diciembre, El Colegio de la Frontera Norte, Tijuana Baja, California.

HIERNAUX, Daniel. 1986. Urbanización y autoconstrucción de vivienda en Tijuana. Centro de Ecodesarrollo, México.

IBARRA, V. y F. Saavedra (compiladores). 1986. "La ciudad y el medio ambiente en América Latina: el caso de la zona metropolitana de la ciudad de México", COLMEX, México.

INEGI, 1991a. XI Censo general de población y vivienda, 1990, México.

- 1991b.XI Censo general de población y vivienda, 1990. Resultados definitivos. Baja California.

- 1992. Avance de Información Económica, Industria maquiladora de exportación.

JÁUREGUI, Emesto. 1981. "Aspectos metereológicos de la contaminación del aire a lo largo de la frontera norte de México". 2da. Reunión Estados Unidos-México sobre estudios de las fronteras, Universidad de Texas. Austin, Tex.

LELE, M. Sharachandra. 1991. "Sustainable Development: A Critical Review", World Development, vol. 19, no. 6, en Negrete Ma. Eugenia, et al. 1993. "Población, espacio y medio ambiente en la zona metropolitana de la ciudad de México". Centro de Estudios Demográficos y de Desarrollo Urbano, El Colegio de México.

Ministerio de Obras Públicas y Urbanismo. 1990. Desarrollo y medio ambiente en América Latina y el caribe. Una visión evolutiva. Madrid.

MORALES, Rafael. 1986. "Evaluación de la contaminación en Playas de Tijuana" en Álvarez, Juan y Víctor Castillo (coordinadores). Ecología y frontera. Escuela de Economía, UABC, México.

PÉREZ, Benigno y Raymundo Reyes. 1986. "Niveles de plomo en La Gloria, B.C." en Juan Álvarez y Víctor M. Castillo (coords.). 
Ecología y Frontera:el estudio del medio ambiente en las Californias, Escuela de Economía, UABC, Tijuana, México.

RANFLA, G. Arturo y Guillermo Álvarez. 1986. "Expansión física, formas urbanas y migración en el desarrollo urbano de Tijuana, 1900-1984." Cuadernos de Ciencias Sociales, serie 3, no. 2, ISUABC. México.

RANFLA, G. Arturo, et al. 1989. "Expansión física y desarrollo urbano de Tijuana 1900-1984", Historia de Tijuana, 1889-1989, UABC y Gobierno del Estado de Baja California.

OJEDA, Lina y Carlos de la Parra. 1989. "Parques y jardines". COLEF (mimeo).

OJEDA, Olga y Vicente Sánchez. 1984. "La cuestión ambiental y la articulación sociedad-naturaleza". Programa de desarrollo y medio ambiente, COLMEX, (mimeo).

SAHOPE. 1984. Plan de Desarrollo de la Ciudad de Tijuana. Mexicali, Baja California.

- 1991. Esquema de Desarrollo Urbano, 1991-1995. Ciudad de Tijuana. XIII Ayuntamiento. Mexicali, Baja California.

SÁNCHEZ, Roberto. 1988. "Conflictos ambientales y negociación binacional entre México y Estados Unidos". Documento de trabajo. Fundación Friedrich Ebert, COLEF.

- 1989. "Contaminación de la industria fronteriza: riesgos para la salud y el medio ambiente". González-Aréchiga, Bernardo y Rocío Barajas Escamilla (compiladores). Las maquiladoras. COLEF y Fundación Friedrich Ebert.

- 1991. "El Tratado de Libre Comercio en América Latina y el medio ambiente de la frontera norte". Frontera Norte, vol. 3, no. 6, julio-diciembre, COLEF. Tijuana, Baja California.

SÁNCHEZ, Vicente, et al. 1982. Glosario de términos sobre medio ambiente. COLMEX., México.

SCHTEINGART, Martha. 1987. "Expansión urbana, conflictos sociales y deterioro ambiental en la ciudad de México. Estudios Demográficos y Urbanos, vol. 2, no. 3, COLMEX.

SEDUE. 1986. Estado del medio ambiente en México. Fundación Arturo Rosenblueth, México.

SOMEDE. 1992. Comentarios del Seminario: "Poblacion y Ambiente". 8-10 de abril, Morelos, México.

TUDELA, Fernando. 1991. "Población y medio ambiente". DEMOS, Carta Demográfica sobre México. México.

TREJO, Vázquez. 1987. "La disposición de desechos sólidos urbanos". Ciencia y Desarrollo, Mayo-junio, CONACYT, México. 


\section{Periódicos:}

\section{Diario 29}

LU, Argelia. 24 de marzo de 1992, Tijuana Baja California.

- 20 de abril de 1992. Tijuana Baja California.

RENTERÍA, Horacio. 11 de enero de 1992. Tijuana, Baja California.

\section{El mexicano}

MARTÍNEZ, Hortencia. 16 de febrero de 1992, Tijuana, B.C. 